\title{
Analysis of the reliability of heat supply from hybrid energy source based on WPP and CHPP
}

\author{
Ivan Postnikov ${ }^{1, *}$ \\ ${ }^{1}$ Melentiev Energy Systems Institute of Siberian Branch of the Russian Academy of Sciences, Laboratory of Heat Supply Systems, \\ 664033 Irkutsk, Russia
}

\begin{abstract}
The paper is devoted to the problem of the reliability analysis the functioning of the hybrid energy source based on the wind power plant (WPP) and the combined heat and power plant (CHPP). The main effect of the proposed technology is substituting a part of the fossil fuel used at CHPP (or other heat sources) for the production of electricity and thermal energy. At the same time, CHPP operates according to the heat schedule, i.e. in accordance with changes in the heat loads of consumers. In this regard, the attention in the study is focused on the problem of the reliability analysis of the heat supply (heating) from a hybrid energy source. To assess the reliability of heat supply, one of the main complex reliability index (RI) is used - the availability factor (AF), and the system of equations of a stationary markov process is used as a mathematical model of the states evolution of the studied object. Calculation of AF is made in accordance with seasonal changes in heat loads (annual heat load schedule); the heat storage effect of consumers (thermal inertia) and the factor of a possible decrease in wind speed are also additionally taken into account. Case study is carried out on a test circuit of the hybrid energy source (WPP + CHPP), as a result of which some analytical characteristics are obtained.
\end{abstract}

\section{Introduction}

One of the possible technologies for the joint operation of the wind power plant (WPP) and the combined heat and power plant (CHPP) is proposed by the authors in the form of a patent for invention [1]. The main idea behind the proposed technology is to use the wind power in the thermal cycle of the heat source (HS) as substituting a part of fossil fuel using for the generation of thermal and/or electricity energy depending on type of HS. For this the electric heating units (EHU) are integrated in the circuit of the HS - boiler plant (BP), CHPP and other types.

Together with fuel-saving and reduction of emissions from CHPP, the technology makes it possible to solve the problem of instability of the electric power coming from WPP. A detailed description of the proposed technology, various schemes of its implementation, possible economic and environmental effects are presented in [2].

The present study is devoted to the development of methods for the reliability analysis of such a hybrid energy source based on WPP and CHPP. The problem statement of reliability analysis is specified with regard to heat output (heating from source), as WPP energy is mainly consumed for heating purposes from HS (for BP this share amounts to $100 \%$ ).

A fairly large-scale review of studies on WPP reliability, covering more than 100 sources, is presented in [3]. The approaches to the reliability analysis of the reliability of WPP based on probabilistic methods and models of state estimation are presented in [4-7]. Much attention is also paid to improving the reliability of WPP. For example, in [8], the authors present a review of methods for increasing the reliability of WPP operation with the use of models of fault detection and fail-safe control. Some other methods in the framework of this issue are discussed in $[9,10]$.

The methods for the reliability analysis of the HS of different types can be divided into analytical and statistical modeling methods. The methods of the first group are based mainly on the application of markov and semi-markov random processes models [11-14]. The methods of the second group are based on the use of simulation algorithms in the framework of which usually statistical test method (Monte Carlo method) is applied to model possible states of the facility $[11,15,16]$.

\section{Methods}

To assess the reliability of heat output (heating) from a hybrid energy source based on WPP and CHPP or other $\mathrm{HS}$, it is proposed to use one of the main comprehensive reliability index (RI), the availability factor (AF) noted as $K$, which can be calculate by the follow formula:

$$
K=1-\sum_{s \in E}\left[p_{s}(1-\omega)^{-\alpha}\left(1-\left(q_{s} / q_{0}\right)\right)^{\alpha}\right]
$$

where $s$ - energy source state numbers (further - states); $E$ - complete set of states; $p_{s}$ - state probabilities; $\alpha, \omega$

*Corresponding author: postnikov@,isem.irk.ru 
- heat load profile non-uniformity coefficients [17]; $q_{s}-$ level of heat output from the energy source in state (taking into account changes in heat loads), MW; $q_{0}-$ design heat load connected to the energy source, MW.

Probabilities of system states is determined by solving the equation system of the stationary markov process describing the evolution of events in the system:

$$
p_{s} \sum_{n \in N_{s}}\left(\lambda_{n}+\mu_{n}\right)=\sum_{z \in E_{s}} p_{z} \sum_{n \in N_{z}}\left(\lambda_{n}+\mu_{n}\right)
$$

where $p_{z}$ - state probabilities (separation of states on $s$ and $z$ is necessary for formulation equation of the markov process); $n$-number of the energy source element; $N_{s}$ - a subset of elements of the energy source, the failure or restoration of which corresponds to a direct transition from state $s$ to state $z ; N_{z}$ - a subset of energy source elements, the failure or restoratin of which corresponds to a direct transition from state $z$ to state $s$; $E_{s}-$ a subset of states from which a direct transition to the state $s ; \lambda_{n}$ - failure rate of the energy source element $n, 1 / \mathrm{h} ; \mu_{n}$ - restoration rate of the energy source element $n, 1 / \mathrm{h}$. Applied of markov models for the solving of reliability problems of HS and district heating reliability is considered in $[11,13,18-20]$.

The expression (1) allow us to determine AF taking into account the changes in heat loads (heat load schedule) by the parameters $p_{s}, \alpha$ and $\omega$, which are members of the Rossander equation [17], that allow us calculate the heat load in any time point as a function of its duration within the calculation (heating) period.

The follow expression allow us calculating AF of hybrid energy source in relation to the heat supply, taking into account the heat storage effect:

$$
\left.K=1-\sum_{s \in E}\left[p_{s}(1-\omega)^{-\alpha}\left(\varphi\left(t_{s}-t_{\mathrm{exs}}\right) / q_{0}\right)\right)^{\alpha}\right]
$$

where $\varphi$ - specific heat consumption coefficient (heating characteristic of consumers), $\mathrm{MW} /{ }^{\circ} \mathrm{C} ; t_{s}$ - indoor air temperature at consumer in state $s,{ }^{\circ} \mathrm{C} ; t_{\mathrm{exs}}$ - outdoor air temperature (outdoor temperature), at which the time redundancy equals the time of restoring the design (standard) heat supply from the emergency state $s,{ }^{\circ} \mathrm{C}$.

The time reserve related heat storage effect in the heating system is factored in by means of the following expression to determine the temperature $t_{\mathrm{exs}}$ [11]:

$$
\begin{gathered}
t_{\mathrm{exs}}=\frac{t_{0}\left(1-\bar{q}_{s}\right)-\left(t_{\min }-t_{0} \bar{q}_{s}\right) \exp B_{s}}{\left(1-\bar{q}_{s}\right)\left(1-\exp B_{s}\right)}, \\
\bar{q}_{s}=q_{s} / q_{0}, B_{s}=\left(\varepsilon \mu_{s}\right)^{-1},
\end{gathered}
$$

where $\bar{q}_{s}$ - relative (as a percentage of the design level) heat output from the energy source in state $s ; t_{\min }-$ minimum allowable internal temperature at the consumer,
${ }^{\circ} \mathrm{C} ; t_{0}$ - design internal temperature at the consumers, ${ }^{\circ} \mathrm{C}$; $\varepsilon$ - heat storage factor of consumers (passive time reserve of consumers), $\mathrm{h} ; \mu_{s}$ - restoration rate for state $s, 1 / \mathrm{h}$.

In expression (3) using (4), just like in (1), for each state, its "failure" part of the calculation period (the period of time during which this state is a failure state) is determined, but at the same time, it is corrected according to the time redundancy that is due to the heat storage effect (thermal inertia).

When analysis the reliability of heat supply from the considered hybrid energy source one should take into account the effect of the wind speed. To this end, we propose a methodological approach which is used at the level of probabilistic modeling of the energy source operation. This method is the introducing the "failure" of a simulation element (SE) into the structure of its states, which occurs with the rate of realization of the simulated external factor (in this case - wind speed).

\section{Brief report on case study}

The given scheme of a hybrid energy source based on the WPP and steam-turbine CHPP is presented in Fig. 1. In the figure, electricity flows (EE) are shown as a dotted line while thermal energy flows (TE) are shown as a solid line. As part of the given scheme, a peak boiler house/plant (denoted as "B" in Fig. 1) operates in parallel with the CHPP, which is typical of many heating systems.

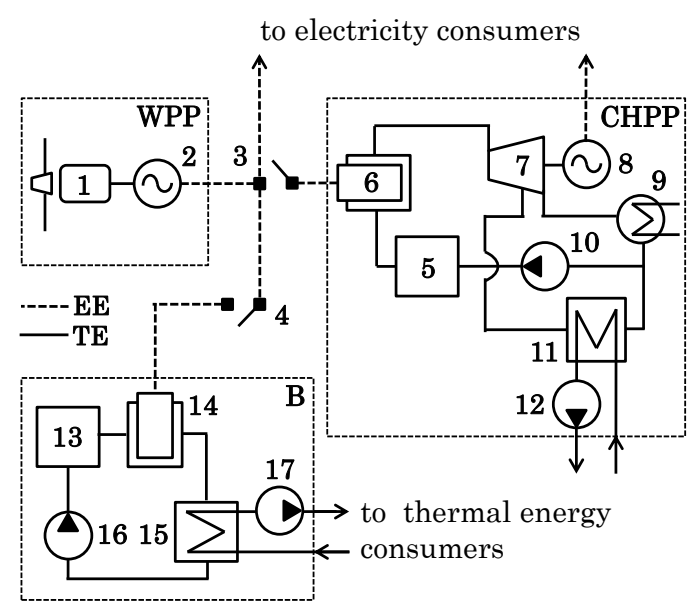

Fig. 1. The schematic of a hybrid energy source based on the WPP and CHPP: 1 - wind turbine; 2 - generator of WPP; 3, 4 switches; 5 - steam boiler of CHPP; 6 - EHU of CHPP; 7 steam turbine; 8 - generator of CHPP; 9 - turbine condenser; 10 - feed pump; 11, 15 - network heaters; 12, 17 - network pumps; 13 - boiler of boiler house/plant (B); 14 - EHU of B.

Three calculation options are considered: option 0 (baseline) - takes no additional factors into account, option 1 - takes into account the heat storage effect, and option 2 - takes into account the heat storage effect and possible wind speed reduction. For all options, the share of the WPP in covering the demand for heat is $50 \%$.

The formation of the set of hybrid power source states is based on a comprehensive considering of WPP, CHPP, and peak B schemes, taking into account joint failures of 
their elements. In this case, the constraint on the simultaneous failure of no more than two elements belonging to different subsystems (WPP, CHPP, and B) is introduced. The graph for such state structure consists of 110 components (vertices), 18 of them correspond to simple states and 92 of them correspond to compound (complex) ones. Each transition between states consists of the realization of only one event, that of failure or restoration of some element (i.e. the event flow path is ordinary).

Taking into account possible wind speed reduction is carried out in line with the approach described in section 2 ; to this end, the SE is introduced into the structure of the energy source states, the failure of this is interpreted as such a wind speed reduction that stops the energy transfer from the WPP to the CHPP. The formed structure of the states of the investigated facility and transitions between them is governed by a random markov process, describing by equation system (2). As a result of solving the obtained system of equations, the probabilities of energy source states are determined.

Table 1 and Fig. 2 present the results of the reliability analysis of heat supply from a hybrid energy source based on the WPP and CHPP for the three calculation options described above.

Table 1. Results of the analysis of reliability of heat supply from a hybrid energy source based on the WPP and CHPP

\begin{tabular}{|c|c|c|c|}
\hline \multicolumn{4}{|c|}{ Availability factor } \\
\hline \multirow{2}{*}{ Subsystem } & 0 & 1 & 2 \\
\cline { 2 - 4 } & \multicolumn{4}{|c|}{ Calculation option } \\
\hline \multicolumn{4}{|c|}{ Comprehensive solution (CS) } \\
\hline \multicolumn{4}{|c|}{ Decomposition } \\
\hline WPP & 0,9954 & 0,9994 & 0,9868 \\
\hline CHPP & 0,9906 & 0,9928 & 0,9928 \\
\hline B & 0,9981 & 0,9998 & 0,9998 \\
\hline
\end{tabular}

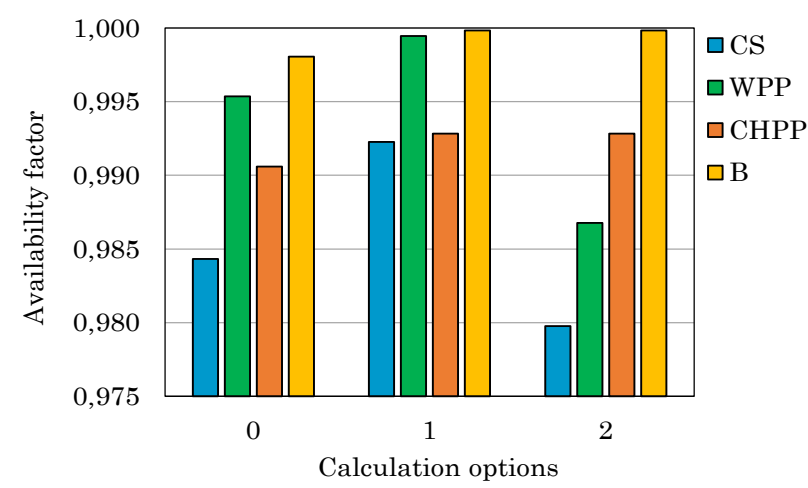

Fig. 2. Results of the analysis of the reliability of a hybrid heat source based on the WPP and CHPP

The AF index is determined both for comprehensive solution (CS) and for the decomposition one. Fig. 2 demonstrate that the highest $\mathrm{AF}$ value corresponds to option 1 that factors in the heat storage effect providing an additional time redundancy. The lowest AF level corresponds to option 3 taking into account the state simulating a decrease in wind speed leading to a shutdown of the WPP power transmission.
A series of additional calculations was carried out to arrive at dependencies between RIs and initial modeling conditions. Variable initial parameters: share of WPP power used in the CHPP cycle and the reliability criterion being minimum allowable internal air temperature at the consumers. Fig. 3 presents a diagram showing AF change depending on the values of two parameters: a percentage of CHPP fuel substitution for WPP power and minimum allowable internal temperature.

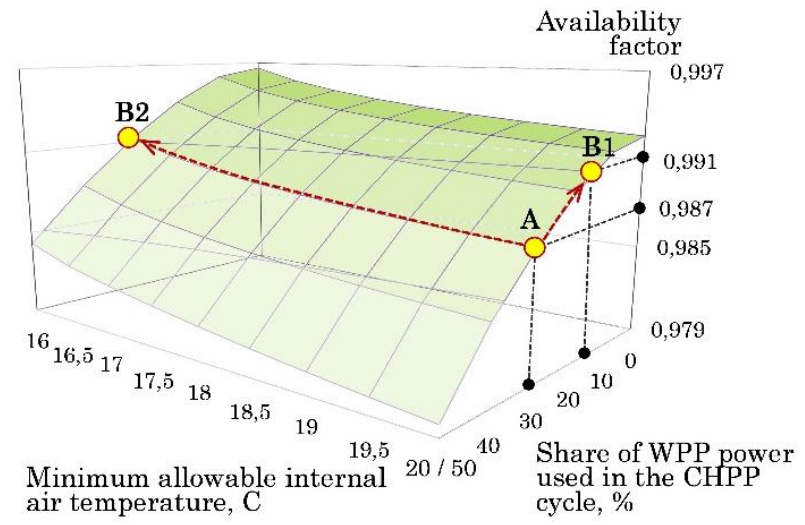

Fig. 3. Change of AF with respect to heat output from a hybrid energy source based on the WPP and CHPP depending on the initial modeling conditions (for calculation option 2)

The diagram in Fig. 3 is shown for substitution share values from the WPP up to $50 \%$ because at higher percentages the AF values are reduced to practically unacceptable levels. It follows from the diagram that AF values increase at a higher rate given a decrease in the share of WPP that given a decrease in requirements for the reliability of heat supply with respect to the temperature criterion. So, for example, given the substitution percentage by WPP equal to $30 \%$, the $\mathrm{AF}$ at the minimum allowed internal temperature of $20^{\circ} \mathrm{C}$ is 0.987 (point $\mathrm{A}$ in Fig. 3). Increasing reliability from this point to the AF value equal to 0.991 is achieved when the share of the WPP use is reduced to about $10 \%$ (point B1) or the reliability criterion is reduced to $16^{\circ} \mathrm{C}$ (point $\mathrm{B} 2$ ).

\section{Conclusions}

Methods and models to analysis the reliability of heat output from a hybrid energy source based on the WPP and CHPP are developed. As an indicator of reliability, we used the availability factor, which is determined factoring in the profile of heat loads during the calculation period (usually the heating period) and the time reserve due to the heat storage effect. Modeling of states of the investigated facility and transitions between them is carried out on the basis of the equations of a stationary markov process. The reliability analysis also takes into account the random factor of wind speed reduction.

Case study is provided including the analysis of the test scheme of a hybrid power source based on the WPP and CHPP and processed and analyzed the obtained results. These results are primarily aimed at testing the developed methods and should not be interpreted as universal. Nevertheless, some qualitative ratios of the 
obtained characteristics reflect general properties for objects of this type of study. For example, the characteristics obtained as a result of the computational experiment reveal the conflict between the environmental effect and reliability: reduction of emissions from the CHPP is proportional to the share of fuel substitution for WPP power, at the same time, when this share increases, the reliability of heat output decreases, which is associated with a possible event of off-design wind speed reduction.

The reported study was funded by RFBR, project number 20-08-00488 A

\section{References}

1. S. Zharkov, A. Kejko, I. Postnikov, A. Penkovskii, Operation of the steam power plant, RU Patent No.2557049 RF (2015)

2. I. Postnikov, V. Stennikov, A. Penkovskii, Energy Procedia 158 (2019)

3. E. Artigao, S. Martín-Martínez, A. HonrubiaEscribano et al., Applied Energy 228 (2018)

4. H. Guo, S. Watson, P. Tavner et al., Reliability Engineering and System Safety 94 (2009)

5. M.M. Slot Rene, J.D. Sørensen, B. Sudret et al., Renewable Energy 151 (2020)

6. X. Zhang, L. Sun, H. Sun, J. Wind Eng. Ind. Aerodyn 154 (2016)

7. S. Eryilmaz, Y. Devrim, Reliability Engineering and System Safety 185 (2019)

8. H. Habibi, I. Howard, S. Simani, Renewable Energy 135 (2019)

9. Y. Zhu, C. Zhu, C. Song et al., Electrical Power and Energy Systems 113 (2019)

10. F. Wang, J. Chen, B. Xu et al., Applied Energy 251 (2019)

11. E. Sennova, A. Smirnov, A. Ionin et al., Reliability of heat supply systems (Nauka, Novosibirsk, 2000)

12. J.-J. Wang, C. Fu, K. Yang et al., Energy 61 (2013)

13. A. Lisnianski, D. Elmakias, B.H. Hanoch, Reliability engineering \& system safety 98 (2012)

14. H. Sabouhi, A. Abbaspour, M. Fotuhi-Firuzabad, P. Dehghanian, International Journal of Electrical Power \& Energy Systems 79 (2016)

15. N. Buslenko, Modeling of complex systems (Nauka, Moscow, 1978)

16. L. Shua, L. Chena, J. Jina et al., Applied Energy 80, 1 (2005)

17. E. Sennova, V. Sidler, Mathematical modeling and optimization of developing heat supply systems (Nauka, Novosibirsk, 1987)

18. I. Postnikov, V. Stennikov, E. Mednikova, A. Penkovskii, Applied Energy 227 (2018)

19. V. Stennikov, I. Postnikov, Power Technology and Engineering 47, 6 (2014)
20. I. Postnikov, V. Stennikov, Energy Reports 6, 2 (2020) 